\title{
Neurobiological Insights into Twice Exceptionality: Circuits, Cells, and Molecules
}

\author{
Authors: Benjamin A. Kelvington ${ }^{1}$, Thomas Nickl-Jockschat ${ }^{2}$, Ted Abel ${ }^{1 *}$ \\ 1: lowa Neuroscience Institute, Carver College of Medicine, University of lowa, lowa City, IA, USA; Department of Neuroscience and \\ Pharmacology, Carver College of Medicine, University of lowa, lowa City, IA, USA.
}

2: lowa Neuroscience Institute, Carver College of Medicine, University of lowa, lowa City, IA, USA; Department of Psychiatry, University of Iowa, lowa City, IA, USA; Department of Neuroscience and Pharmacology, Carver College of Medicine, University of lowa, lowa City, IA, USA.

*Corresponding author at: University of lowa, 169 Newton Road, lowa City, IA, USA.

Email address: ted-abel@uiowa.edu

Abstract: Twice-exceptional learners face a unique set of challenges arising from the intersection of extraordinary talent and disability. Neurobiology research has the capacity to complement pedagogical research and provide support for twiceexceptional learners. Very few studies have attempted to specifically address the neurobiological underpinnings of twiceexceptionality. However, neurobiologists have built a broad base of knowledge in nervous system function spanning from the level of neural circuits to the molecular basis of behavior. It is known that distinct neural circuits mediate different neural functions, which suggests that $2 e$ learning may result from enhancement in one circuit and disruption in another. Neural circuits are known to adapt and change in response to experience, a cellular process known as neuroplasticity. Plasticity is controlled by a bidirectional connection between the synapse, where neural signals are received, and the nucleus, where regulated gene expression can return to alter synaptic function. Complex molecular mechanisms compose this connection in distinct neural circuits, and genetic alterations in these mechanisms are associated with both memory enhancements and psychiatric disorder. Understanding the consequences of these changes at the molecular, cellular, and circuit levels will provide critical insights into the neurobiological bases of twice-exceptional learning.

Keywords: Twice-exceptionality, 2e, Memory Systems, Plasticity, Epigenetics

\section{Introduction}

Twice-exceptional (2e) learners are students who possess extraordinary cognitive abilities, or demonstrate a capacity for high achievement, in one or more academic areas, while also facing challenges in the form of a diagnosed disability (Reis, Baum, \& Burke, 2014). 2e is a highly heterogenous condition encompassing a variety of strengths and psychiatric disorders. One study of $2 e$ incidence estimates that roughly 360,000 students in the U.S. meet the criteria for $2 \mathrm{e}$, which correlates to roughly $0.7 \%$ of all $\mathrm{K}-12$ students (National Education Association, 2006). 2e learners face unique challenges that result from the intersection of their increased ability and unique disability (Assouline, Nicpon, \& Huber, 2006). As a result, many $2 \mathrm{e}$ learners are not sufficiently supported by traditional special education and/or gifted education (Assouline \& Whiteman, 2011; Beckmann \& Minnaert, 2018). Pedagogical research has dominated the field of $2 \mathrm{e}$ as experts try to determine how best to meet the needs of $2 \mathrm{e}$ students (Foley-Nicpon,
Assouline, \& Colangelo, 2013). A few studies have attempted to characterize the cognitive profiles of a variety of $2 \mathrm{e}$ cohorts, where strengths are indicated in some areas and relative deficits in others (Budding \& Chidekel, 2012; Maddocks, 2019; Cain, Kaboski, \& Gilger, 2019). Furthermore, multiple psychological theories have been proposed to explain relative intellectual strengths and weaknesses within an individual (Gardner, 1983; Sternberg, 1985). However, this literature does little to address brain function in individuals with $2 \mathrm{e}$.

A more complete understanding of the neural mechanisms underlying 2 e could provide insights that help to both promote the achievement of $2 \mathrm{e}$ learners by identifying their strengths as well as provide necessary support in areas of struggle (Gilger \& Hynd, 2008). A candidate to guide the neurobiological study of $2 e$ is plasticity. Plasticity is hypothesized to explain the basis of how the brain responds to experience and stores memory (reviewed in Takeuchi, Duszkiewicz, \& Morris, 2014). Plasticity has been linked to intellectual giftedness (Brans et 
al., 2010), and dysregulation of plasticity is implicated in neurodevelopmental disorders such as autism spectrum disorder (ASD) (Bourgeron, 2015; Hansel, 2019). The knowledge and investigative techniques developed by neuroscientists to study neuroplasticity have the power to delineate the changes that result in many of the variable presentations of $2 \mathrm{e}$. The investigation of changes in plasticity at the circuit level may reveal how some neural functions become enhanced in $2 \mathrm{e}$ while others are disrupted. Investigation at the cellular and molecular levels may illuminate the neurobiological underpinnings of these 2e phenotypes. Such investigations will have wide-ranging implications for the support of $2 \mathrm{e}$ learners as well as our broader understanding of the brain.

\section{Behavior is Mediated by Distinct Neural Circuits}

\subsection{Memory Systems}

The neurobiological study of $2 \mathrm{e}$ will require an understanding of how distinct neural functions are affected in a given $2 \mathrm{e}$ phenotype. The multiple memory systems (MMS) hypothesis has dominated the contemporary understanding of how distinct neural functions are mediated, and it provides a promising framework for the explanation of 2e phenotypes (Sherry \& Schacter, 1987; Squire, 1992). MMS postulates that information is stored in the brain via distinct and independent modules that mediate unique aspects of memory. Memory is the maintenance of learning, experience-dependent changes in behavior, over time. These modules manifest as distinct neural circuits, each with their own structural and functional properties. The most convincing support for MMS comes from lesion studies, where memory is studied in humans or other model organisms with selective damage to a specific module (Figure 1). The seminal, and most famous, lesion study is that of H.M., a patient with bilateral lesions of the hippocampus (Scoville \& Milner, 1957). H.M. was unable to establish new declarative memories, which refers to his inability to remember facts and events. Importantly, H.M.'s memory deficits largely did not translate to declarative memories acquired prior to hippocampal lesioning or the learning of new motor skills, known as procedural memory. In other words, H.M. could learn new motor skills, but he could not remember the circumstances in which he learned them. More recent lesion studies have provided further support to the MMS hypothesis and dissociated the memory functions of other brain regions such as the amygdala and dorsal striatum (McDonald \& White, 1993; Bechara et al., 1995). One such study was able to dissociate the memory functions of the hippocampus and amygdala in human patients with selective lesions to each region using a classical conditioning paradigm (Bechara et al., 1995). The participant with selective hippocampal lesions, like H.M., was unable to recite declarative facts about the conditioning experience but still acquired the conditioned physiologic response as measured by skin galvanic response (Figure $1 \mathrm{~A}$ ). The participant with selective lesions of the amygdala, on the other hand, did not acquire the conditioned physiologic response but could answer questions about the experience indicating the formation of episodic memory (Figure 1B). These lesion studies support the idea that different functions are mediated by independent neural modules with distinct underlying mechanisms, and disruption of a module also disrupts the function that it controls. 2e phenotypes may then plausibly result from disruption in one system coexisting with enhancement in another system.

\subsection{Interactions between Memory Systems}

Although the MMS hypothesis continues to accurately explain the nature of unique and independent facets of memory, recent data has led to slight variations in MMS theory that account for the complex crosstalk between memory systems (Ferbinteanu, 2019). The basis of this variation in MMS is that memory formation in real-world settings can be more complex than what is measured in controlled laboratory experiments. This complexity necessitates the involvement of multiple memory systems whose usually parallel functions interact to support memory

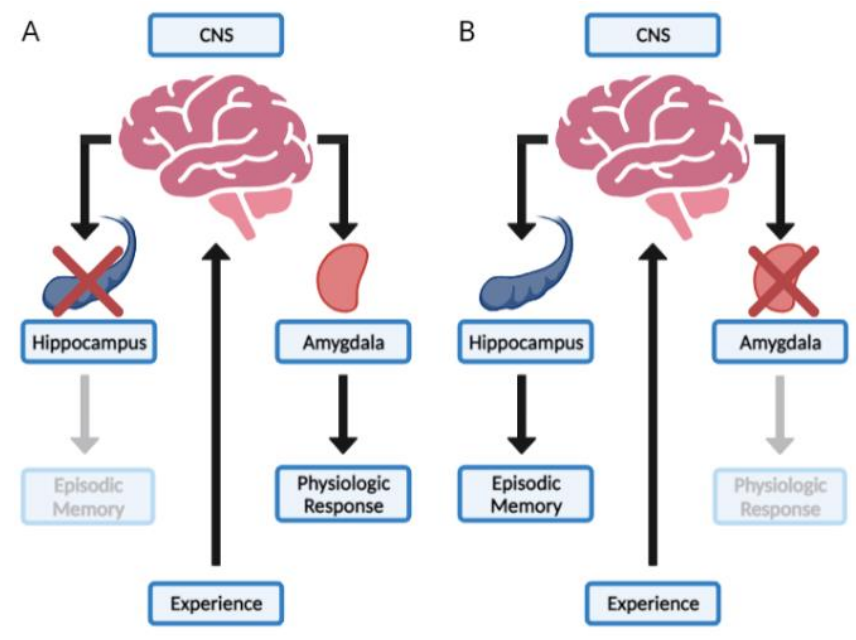

Figure 1. Double Dissociation of Memory Systems. A) Lesioning of the hippocampus disrupts episodic memory formation, while leaving the physiologic response intact. B) Lesioning of the amygdala prevents the physiologic response, while episodic memory formation is preserved. 
formation. Lesion experiments support that some memory functions are the result of interactions between memory systems (McDonald \& White, 1995). Interactions are revealed when the lesioning of one memory system induces a different memory system to produce the same memory function, or the lesioning of both systems is required for the loss of memory function. These interactions may explain the ability for a memory system that is secondary to a given behavior to compensate for the dysfunction of the dominant memory system based upon the needs of the organism. Network neuroscience employs graph theory to attempt to map these complex and dynamic interactions between memory modules (Bassett \& Sporns, 2017). This is just one approach by which scientists are beginning to address the challenge of characterizing the neural circuitry involved in complex behaviors such as learning and memory, and more work needs to be done to fully understand these interactions. However, an understanding of neural circuits as dynamic and capable of compensating for one another is essential to the study of learning and memory in general and in the context of $2 e$.

\subsection{Candidate Circuits in $2 e$}

Although the specific neural circuits underlying $2 \mathrm{e}$ have yet to be delineated, a wide range of circuits have been suggested to be involved in various $2 e$ phenotypes. For example, the prediction impairment in autism (PIA) hypothesis, which posits that ASD results from the dysfunction of predictive circuits, implicates the basal ganglia in general and the striatum more specifically (Sinha et al., 2014; see also Nickl-Jockschat et al., 2012; Janouschek et al., 2021). Under this hypothesis 2e-ASD is thought to result in exceptional ability in certain disciplines, such as mathematics, where strict rules exist and prediction is less vital. Another interesting hypothesis proposes that declarative memory systems may act to compensate for dysfunction in other memory systems in the context of a variety of psychiatric conditions (Ullman \& Pullman, 2015). The declarative memory compensation hypothesis postulates that the declarative memory systems of the medial temporal lobe (MTL), in which the hippocampus is the major player, can make up for deficits in other memory systems. This idea is supported by the observation that declarative memory remains largely intact across several neurodevelopmental disorders including ASD (Boucher \& Mayes, 2012; Boucher, Mayes, \& Bigham, 2012). Furthermore, one study suggests that individuals with ASD may use declarative memory dependent strategies, such as memorizing social scripts, to overcome social challenges (Portman, 2006). Another imaging study showed hypoactivation of the nucleus accumbens (basal ganglia) and hyperactivation of the hippocampus during a social reward anticipation task, which suggests that declarative memory could be compensating for a critical deficit in the basal ganglia circuitry (Dichter et al., 2011). This hypothesis might help to explain the experience of many $2 \mathrm{e}$ individuals where their specific disability is masked by extraordinary abilities in other domains such as declarative memory. Similarly, the hyper systemizing theory of ASD, which hypothesizes that individuals with ASD are better able to identify the regular causal rules of an input-output system, has implicated cortical regions involved in sensory processing and perception including the lateral frontoparietal circuity (Baron-Cohen, 2006; Baron-Cohen \& Lombardo, 2017). The same theory also suggests that motivation is a critical component of 2e-ASD, which points to corticostriatal circuits and their interaction with the dopamine system. Importantly, under each hypothesis it is proposed that the same underlying mechanisms that lead to ASD also create the opportunity for exceptional ability. It is also important to remember that although most of the work regarding the neural basis of 2 e focuses on $2 e-A S D, 2 e$ can involve a wide variety of psychiatric conditions, each of which may involve a unique neurobiological etiology. Therefore, although the proposed circuit differences serve as a promising starting point for the neurobiological investigation of $2 e$, specific $2 e$ phenotypes will require emphasis on unique and varied neural circuits.

\section{Plasticity Controls a Circuit's Response to Experience}

\subsection{Synaptic Plasticity}

A challenge for the field of neuroscience research for more than a century has been to describe the cellular basis of how neural circuits are altered in response to experience and how these changes persist to influence behavior. The leading explanation of this phenomenon is termed synaptic plasticity, which was first proposed by Donald Hebb in 1949 when he observed that the sequential activation of neurons creates a strengthening of the synaptic connection between them (Hebb, 1949). Many avenues of research in recent decades have continued to support, strengthen, and add complexity to Hebb's original findings, which has led to the creation and refinement of the synaptic plasticity in memory (SPM) hypothesis (Takeuchi, Duszkiewicz, \& Morris, 2014). The central postulation of SPM is that activity-dependent synaptic plasticity at specific synapses following an experience is both necessary and sufficient to produce memory. Synaptic plasticity can manifest as the strengthening of synaptic connections through an increase in synaptic efficiency, which is known as long-term potentiation (LTP). LTP is the form of plasticity most closely associated with memory formation in response to experience. On the other hand, plasticity could also entail 
the weakening of synapses, known as long-term depression (LTD). Plasticity may also include the addition of new synapses or the removal of existing synapses. Another key provision of the SPM hypothesis is that activity-dependent plasticity mediates the storage of information that is specific to the neural module where it occurs (Martin, Grimwood, \& Morris, 2000). This provision accommodates the MMS hypothesis and provides a theoretical framework for the study of synaptic plasticity in relation to $2 \mathrm{e}$, where enhanced plasticity in one memory system results in extraordinary ability, while disrupted plasticity in another memory system underlies disorder.

\subsection{Measuring Plasticity}

The study of LTP in animal models is a critical tool for researchers in the fields of learning and memory. The field of LTP began in 1973 when Terje Lømo reported a stable increase in synaptic strength in the hippocampus of a rabbit after providing high-frequency electrical stimulation to presynaptic axons (Bliss \& Lømo, 1973). This study provided the first physical evidence for the plasticity of neuronal connections that was first postulated by Santiago Ramón y Cajal more than a century ago (Ramón y Cajal \& Azoulay, 1894). Today, LTP is generally categorized into two distinct phases: early LTP (E-LTP) and late LTP (L-LTP) (Baltaci, Mogulkoc, \& Baltaci, 2018). These phases are separated both temporally, as E-LTP occurs prior to L-LTP, and biochemically, where L-LTP is dependent upon the transcription and translation of new proteins while E-LTP is translation independent. Stable L-LTP is believed to be more relevant to long-term memory formation and hence will be the focus of this review. Experiments that study animal learning behavior while targeting the NMDA receptor, which is critical for hippocampal LTP, provide a direct link between LTP and learning (Morris et al., 1986; Giese et al. 1998). Today, LTP experiments provide neuroscientists the ability to explore synaptic plasticity in defined circuits and under controlled experimental conditions. In particular, ex vivo recordings of mouse hippocampal slices allow for the study of LTP responses and provide a cellular model of learning (Figure 2). Researchers have used this technique to define how various genetic changes lead to alterations in LTP. Notably, genetic studies have demonstrated the role for the molecule PKA in the late phase of LTP in the CA1 region of the hippocampus (Abel et al., 1997). Moreover, although LTP experiments are most well-defined within the tri-synaptic circuit of the hippocampus, LTP has also been observed in other circuits, such as the those in the amygdala and striatum (Nabavi et al, 2014; Lovinger, 2010). Importantly, LTP at synapses in these different circuits can have distinct properties and disparate underlying mechanisms. These differences provide an opportunity for LTP in some circuits to become enhanced while others may be disrupted under the same conditions. Therefore, the utilization of LTP experiments to study plasticity in different neural circuits provides a promising investigative angle to interrogate the MMS hypothesis of $2 \mathrm{e}$.

\subsection{Plasticity and $2 e$}

There is a lack of evidence to indicate specifically the roles that plasticity may play in 2e. However, the SPM hypothesis provides a promising framework for the investigation of $2 \mathrm{e}$ phenotypes. For instance, one could imagine that plasticity mechanisms in the hippocampus could be enhanced in an individual, while plasticity in the basal ganglia may be disrupted in that same individual. The result might then be a 2e phenotype where the prediction or motivation circuity is disrupted, while declarative memory is enhanced. Based upon the nature of the memory systems involved, the same underlying mechanisms may be responsible for both the enhancement and disruption of a given system. Alternatively, distinct mechanisms may create disparate functional outcomes for each system. Complicating this
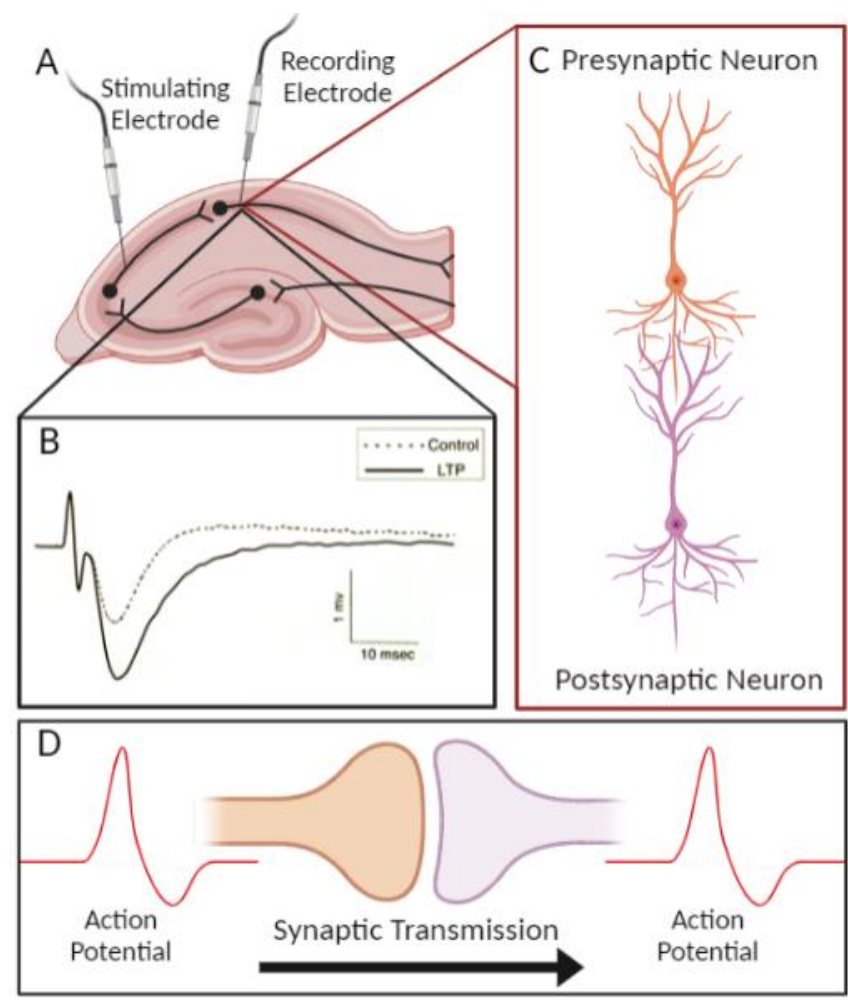

Figure 2. Hippocampal LTP is a cellular model of learning. A) Electrophysiological recordings from mouse hippocampal slices provide an established method for measuring LTP ex vivo. B) High frequency stimulation results in LTP as evidenced by a higher magnitude field potential. $C$ and $D$ ) LTP facilitates enhanced communication between the presynaptic and postsynaptic neuron. 
picture is how plasticity in one system, such as declarative memory, may compensate for disrupted plasticity in another (Ullman \& Pullman, 2015). Indeed, the complex ways in which memory systems interact and may functionally compensate for one another are just beginning to be understood (Ferbinteanu, 2019). On the other hand, it is known that the human brain is capable of extraordinary feats in plasticity, especially during early development. For example, certain forms of intractable epilepsy in children is treated with hemispherectomy, which involves the disconnection and removal of an entire cerebral hemisphere (Kim et al., 2018). Remarkably, this treatment is effective in relieving seizures and leaves cognitive functioning generally intact (Kliemann et al., 2021). The plasticity that allows the brain to maintain function in the absence of an entire hemisphere is not fully understood. However, these cases demonstrate the truly extraordinary potential for plasticity in the brain. Therefore, the concept of plasticity as a hallmark of neural function should guide the investigation into 2e phenotypes.

\section{Molecular Mechanisms Mediating Plasticity}

\subsection{The Synapse and Nucleus: A Bidirectional Connection Controls Plasticity}

Behavioral assays of memory, LTP experiments, and studies of synaptic plasticity at the cellular level have all paved the way for a molecular understanding of synaptic plasticity. Molecular studies of plasticity have guided an extensive body of work defining the molecular bases of synaptic plasticity at glutamatergic synapses, as well as provided pivotal insight into the underpinnings of psychiatric disorders. These studies have illuminated a remarkable connection between genes involved in both synaptic plasticity and neurodevelopmental disorders: they function either at the synapse or in the nucleus (Kandel, 2001) (Figure 3 ). The genes that function in the nucleus generally then alter the expression of genes that function at the synapse. This remarkable connection between the synapse and nucleus forms the basis of the neuron's ability to respond to the environment, which constitutes learning. One wellstudied example of this connection is that of the cAMP response element binding protein (CREB) (Abel \& Lattal, 2001; Kandel, 2012). CREB acts as a molecular switch that can be turned on following stimulation by a class of molecules known as kinases, which catalyze phosphorylation of CREB, a common post-translational modification (PTM) used to modify molecular structure and function. These kinases are themselves activated by neuronal stimulation at glutamatergic synapses which can result in increased $\mathrm{Ca}^{2+}$ influx via NMDA receptors and/or increased CAMP resulting from the activation of neuromodulatory receptors. Activated CREB then binds to certain DNA sequences in the nucleus to promote expression of effector genes that function to promote synaptic plasticity. These subsequent stable molecular changes in the neuron that allow for retention of this synaptic response over time is believed to constitute memory storage. Therefore, it is likely that within these molecular mechanisms of memory storage lie the clues to explaining various $2 e$ phenotypes. Molecular variations may lead to enhanced function in the context of one memory system and simultaneously produce disability when applied to another memory system.

\subsection{Epigenetics Mediate Plasticity}

Epigenetic modifications are biochemical reactions that alter gene expression by modifying the structure of chromatin, which is made up of DNA and class of proteins called histones. They are classified into two main categories: histone tail modifications and DNA methylation, although other classes of epigenetic modifications are also important regulators of gene expression. These epigenetic modifications provide the most widely accepted explanation for the molecular basis of memory storage (Levenson \& Sweatt, 2005; Zovkic, 2021). Epigenetic modifications can either loosen the association between DNA and histones, thereby increasing gene expression, or tighten this relationship to limit gene expression. Increased expression of certain genes as a result of relaxed chromatin promotes memory formation, while more compact chromatin generally has the opposite effect (Fischer et al., 2007). Epigenetic modifications are "written" by enzymes that regulate memory and gene expression. One prominent example of such a regulator is CREB, whose neuronal stimulation-dependent activation recruits the transcriptional co-activator CREB-binding protein (CBP) (Kwok et al., 1994). CBP contains intrinsic histone acetyltransferase (HAT) activity, which catalyzes a transfer of an acetyl group onto lysine residues of the histone tail. This epigenetic modification is associated with an open chromatin state and increased gene expression (Gräff \& Tsai, 2013). This CREB-dependent chromatin modification can be either transient or incredibly stable depending upon the cellular circumstances. Importantly, CREB function has been show in mice to be critical for both LTP and long-term memory formation (Guan et al., 2002; Bourtchuladze et al., 1994). Whereas CREB-mediated histone acetylation 
generally promotes gene expression and memory formation, other epigenetic modifications such as histone methylation can oppose this action (Jarome \& Lubin, 2013). For instance, histone methylation at residues such as lysines 9 and 27 of histone 3 generally serve to repress gene expression, where acetylation at these residues increases expression. Histone methylation has also been shown to occur in response to fear conditioning (Gupta et al., 2010). Evidence suggests that the precisely regulated methylation that represses the expression of certain genes while promoting the expression of others is critical for learning (Gupta-Agarwal et al., 2012). Overall, the evidence suggests that strictly regulated epigenetic modifications in response to experience are essential for appropriate learning and memory. Conversely, disruption in epigenetic mechanisms is associated with neurodevelopmental disorders (Peixoto \& Abel, 2012; Siu \& Weksberg, 2017; Christopher, Kyle, \& Katz, 2017). Thus, precise molecular alterations in epigenetic mechanisms may provide key insight into memory enhancements and disorder in $2 e$.

\subsection{Molecular Mechanisms Can Enhance Memory}

Our understanding of the molecular mechanisms underlying memory may provide key insights into many forms of 2e. An
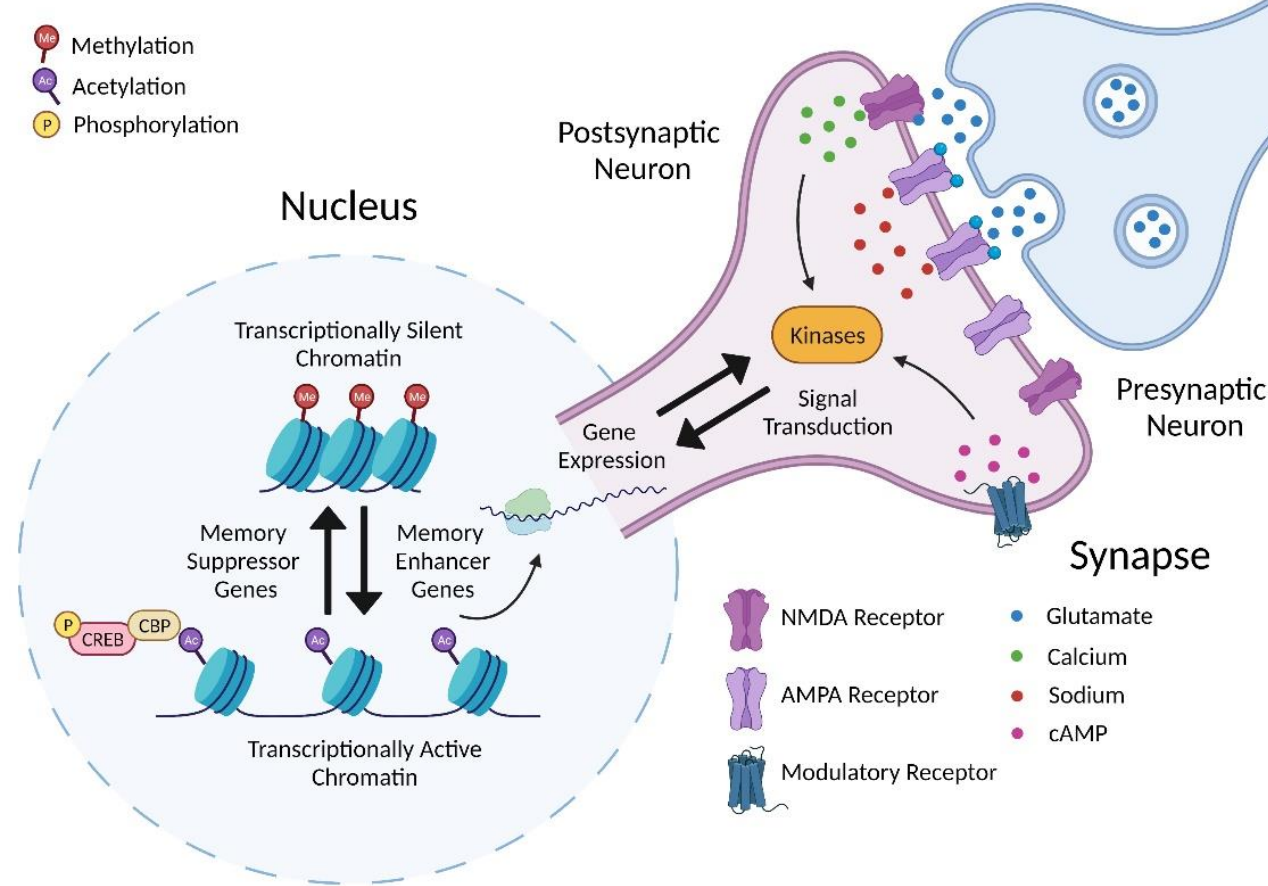

Figure 3. Molecular mechanisms of synaptic plasticity bridge the synapse and nucleus. A series of molecular events spanning the nucleus and synapse result in plasticity at glutamatergic synapses. Neuronal stimulation leads to a postsynaptic influx of $\mathrm{Ca}^{2+}$ and $\mathrm{CAMP}$, which stimulate kinases that signal to the nucleus. Chromatin remodeling in the nucleus changes patterns in gene expression that return to alter synaptic function. interesting mechanism to consider in $2 \mathrm{e}$ is the role of memory suppressor genes (Abel et al., 1998; Noyes, Phan, \& Davis, 2021). Memory suppressor genes, as an analogy to the tumor suppressor genes studied in cancer biology, are those genes whose product functions to inhibit the formation of memories. The existence of such genes is evidenced by the fact that not only can we manipulate memory systems to reduce expression of memory, but we can also enhance the expression of memory through genetic and pharmacological targeting of certain genes (Lee \& Silva, 2009). For example, inhibition of the histone deacetylase (HDAC) enzyme class has been shown to promote long-term memory formation (Burns \& Gräff, 2021). HDACs catalyze the removal of acetyl groups from histone tails, effectively undoing the epigenetic modifications produced by HATs. The typical function of HDACs is to mediate the repression of gene transcription and thereby suppress memory formation. Inhibition of the HDAC enzyme is analogous to "cutting the breaks" on memory formation, allowing for unrestrained memory formation. Similarly, inhibition of the phosphodiesterase family of enzymes, which act to metabolize the key memory effectors cAMP and cGMP, has been shown to enhance hippocampal slice LTP and memory performance in rodents (Boess et al., 2004; van der Staay et al., 2008). Beyond inhibiting memory suppressor genes, other molecular mechanisms can lead to enhancements in learning and memory. Notably, increased expression of an NMDA receptor subunit in the forebrain has resulted in learning enhancements in mice (Tang et al., 1999). These mechanisms of enhanced memory formation have the potential to illuminate the underpinnings of the gifted portion of the 2e phenotype.

\subsection{Molecular 2e: A Case Study of CREB}

To address the more challenging component of $2 \mathrm{e}$, it may be helpful to return to the multiple memory systems hypothesis and consider CREB as an example. The role of CREB in complex behaviors 
such as memory can be difficult to delineate due to the exceedingly complex regulation of CREB activation and CREB-mediated gene transcription in various brain regions and cell types (Carlezon Jr et al., 2005). For example, increased CREB activation is generally associated with enhanced declarative memory formation. One landmark study showed in fruit flies that expression of an activated form of CREB facilitated long-term memory (Yin et al., 1995). $A$ more recent study showed that expression of a mutant CREB that lacks the ability to bind CBP inhibits the formation of long-term memory in mice (Chatterjee et al., 2020). However, increased CREB activation is not universally associated with positive outcomes. Excessive CREB activity in the nucleus accumbens (NAC), a striatal subregion that plays a major role in the reward system, reduces the rewarding feeling associated with sucrose, which indicates a depression-like phenotype (Barrot et al., 2002). Increased CREB phosphorylation in the NAc is also associated with anxiety-like behaviors (Barrot et al., 2005). These data indicate that the effects of CREB activation on behavior depend upon the brain region where the activation is occurring. Therefore, the same genetically determined increase in CREB activation may lead to enhanced hippocampal-dependent memory formation as well as disability resulting from striatal dysfunction. Although intensive study would be needed to confirm such a dissociation of CREB-mediated behavioral effects in a $2 e$ model system, the CREB case study does provide an intriguing framework for the study of 2e. Gaining meaningful knowledge of $2 e$ phenotypes will undoubtedly require the study of molecular mechanisms beyond CREB. However, the hypothesis that distinct behavioral outcomes result from complex molecular interactions in distinct brain regions provides a promising perspective from which to investigate $2 e$.

\section{A Neurobiological Future for $2 e$}

\section{$5.12 e$ Model Systems}

One major obstacle impeding the biomedical investigation of $2 \mathrm{e}$ is a lack of appropriate model systems to study. Therefore, the development and characterization of animal models with relevance to $2 e$ is essential. The primary candidate model system for the study of $2 e$ is the mouse, Mus musculus. Mice have become the primary model system for the study of complex behaviors such as memory due to their well-defined behavioral profile, easily manipulatable genetics, and neuroanatomical similarity to humans (Bućan \& Abel, 2002; Havekes \& Abel, 2009). Mouse models allow researchers to thoroughly study the connection between targeted genetic changes and specific molecular functions, neural circuits, and behaviors that cannot be accomplished in humans. Mouse models with relevance to $2 \mathrm{e}$ could possess a wide variety of genetic manipulations, but they should satisfy two basic behavioral criteria: at least one behavioral indicator of enhanced cognitive ability and at least one behavioral phenotype relevant to a psychiatric disorder. Such a model would allow for the investigation of both behavioral phenotypes and their underlying molecular mechanisms with a focus on disassociating the effects on distinct memory systems. One limitation of studying mouse models is that it can be difficult to develop valid models that reproduce the complex molecular and behavioral presentations of neurodevelopmental conditions in humans (Silverman et al., 2010; Sjoberg, 2017). Indeed, the goal of mouse models is not to replicate the experience of a child with a neurodevelopmental disorder or $2 \mathrm{e}$. Rather, mouse models allow researchers to uncover the molecular underpinnings of neural circuits and behaviors that may be homologous enough to inform our understanding of $2 \mathrm{e}$.

\subsection{Established Model Systems}

Further work is required to define how established molecular mechanisms, such as CREB activation, behave in the context of distinct neural circuits. It is plausible that existing mouse models of neurodevelopmental disorders exhibit behavioral, cellular, or molecular phenotypes that are relevant to $2 \mathrm{e}$, but these phenotypes may go unreported or underappreciated due to their perceived lack of relevance to any neurodevelopmental disorder. Such a phenomenon would represent a variation of the file drawer problem, a bias in scientific publication where non-significant results, or results perceived to be irrelevant to a research question, tend to go unreported (Kennedy, 2004). In this case a strict focus on the identification and treatment of specific deficits may hinder the overall knowledge and clinical benefits produced by neuroscience. Instead, increased attention to results that may indicate enhanced performance, as well as those that are relevant to disorders, will improve our understanding of brain function with respect to both improved performance and neurodevelopmental disorder. Such a shift in orientation will help the neuroscientific community to cultivate an increased understanding and appreciation for both the strengths and struggles of individuals with $2 \mathrm{e}$.

\subsubsection{Setd5 $5^{+/}$: a potential model mouse candidate for $2 e$}


At least one genetic mouse model exhibits the promising characteristics to suggest it has relevance to $2 \mathrm{e}$. The Setd5 haploinsufficiency $\left(\right.$ Setd $5^{+/}$) mouse model, a model of neurodevelopmental disorder that is missing one copy of the Setd5 gene, satisfies our stated criteria for relevance to $2 \mathrm{e}$. The Setd5 protein is an epigenetic regulator that alters the transcription of target genes by its intrinsic histone methylase activity and by regulating acetylation (Kuechler et al., 2014). Gene expression analysis of Setd5 $5^{+/}$mice following contextual fear conditioning shows that Setd5 regulates the expression of several genes whose product acts at the synapse including Neuroligin3 and Shank1 (Deliu et al., 2018). Therefore, Setd5 fits the description of a gene whose product modifies the vital relationship between the synapse and nucleus in response to experience. Furthermore, Setd5 $5^{+/}$mice exhibit multiple behaviors relevant to neurodevelopmental disorder. One striking example is an observed reduction in time that Setd $5^{+/-}$mice tend to interact with a novel mouse (Sessa et al., 2019). This behavioral paradigm is interpreted as a test of social interaction and is used as an indicator of an ASD-like phenotype. Remarkably, this model of neurodevelopmental disorder also exhibits characteristics that suggest its relevance to the gifted component of the $2 \mathrm{e}$ phenotype. Setd $5^{+/-}$mice exhibit enhanced LTP in hippocampal slices and increased long-term memory as measured by contextual fear conditioning and novel object recognition behavioral paradigms (Deliu et al., 2018). Interestingly, the enhancement in fear memory was only seen in male mice when a weaker induction protocol was used, which suggests that protocol variations may reveal memory enhancements in other models as well. This remarkable enhancement of declarative memory in a mouse model of neurodevelopmental disorder provides an exciting opportunity to study the seemingly disparate behavioral phenotypes that result from the same underlying genetic change. Although further study is required to delineate the effects of Setd5 haploinsufficiency in different brain regions or memory systems, existing data indicate that this mouse model represents a promising direction for the neurobiological investigation of $2 e$.

\subsection{Human Studies}

\subsubsection{Neuroimaging}

In addition to studies of 2e-relevant animal models, studies of humans with a specific focus on $2 \mathrm{e}$ will also benefit our understanding of the talents and trials of individuals with $2 \mathrm{e}$. Multiple recording and imaging techniques that can be applied to human subjects have the potential to provide insight into 2e phenotypes. Techniques such as electroencephalogram (EEG) and magnetic resonance imaging (MRI) have been extensively used to study brain activity and structure in relation to human behavior and neurodevelopmental disorder, although few studies exist that specifically address individuals with $2 \mathrm{e}$. However, one such study using functional magnetic resonance imaging (fMRI) showed that an ASD cohort performed similarly to a typically developing cohort on an episodic memory task despite a decrease in functional connectivity between the MTL and the posterior medial network (PM), which is important to performing such tasks (Hogeveen et al., 2020). Their data further suggests that an increase in recruitment of the hippocampus compensates for a decrease in functional connectivity. This finding also provides support for the hypothesis that hippocampal function can compensate for the disruption of certain deficits in $2 e$ learners. Furthermore, a study using EEG, which utilizes electrodes to record electrical activity in the cortex of awake and behaving subjects, found that one $2 \mathrm{e}$ cohort had increased visual perception processing and decreased frontal lobe activity when compared to controls (Bireley, Languis \& Williamson, 1992). These techniques have the power to link certain behavior and performance on cognitive tasks to patterns of neural activity and specific brain regions in individuals, which will serve to connect individual neural circuits to exceptional behavior in 2 e learners.

\subsubsection{Genetic Studies}

Although traditional neuroimaging is a powerful technique for studying brain function, it is limited in describing the genetic underpinnings of $2 \mathrm{e}$. However, other strategies exist that allow the neurobiologist to investigate these genetic contributions in human subjects and samples. For example, large-scale genetic studies such as SPARK, which studies over 50,000 families with at least one ASD diagnosis and maintains an interest in $2 \mathrm{e}-\mathrm{ASD}$, has the power to illuminate some of the genetic underpinnings of 2e-ASD (SPARK Consortium, 2018). Another option is to analyze neuroimaging data, from humans or animal models, alongside available gene expression data sets to connect certain genes to anatomical changes in the brain (Kumar et al., 2018; Richiardi et al, 2015). These techniques provide a powerful tool to implicate specific genes underlying $2 \mathrm{e}$, but they do not allow for the detailed study of gene functions that may comprise the molecular etiology of 2e.

\subsubsection{Studies of Human-Derived Samples}


Molecular mechanisms underlying 2e may be effectively studied in human-derived samples. One promising approach to study molecular mechanisms in human cells is to use induced pluripotent stem cells (iPSCs). iPSCs provide an encouraging avenue for the study of rare or genetically complex conditions (Freel, Sheets, \& Francis, 2020; Drakulic et al., 2020). iPSCs are stem cells that can be harmlessly derived from the skin or blood of an individual with $2 \mathrm{e}$. These cells, which carry the genetic signature of the person they are derived from, can then be differentiated into a range of neural cell types. iPSCs allow for the in vitro study of cellular and molecular function in neural cells with a human genetic profile. A final possible strategy to study the molecular contributions to $2 e$ is the use of post-mortem tissue samples, which can provide a snapshot of the molecular profile of $2 e$ in the human brain. Each of these investigative strategies contributes a piece of the puzzle which, alongside data collected from animal models, will provide critical insight into the molecular underpinnings of $2 e$.

Declarations of interest: none

Funding: This work was supported by the National Institutes of Health [NIH T32 GM067795]

These funding sources played no role in the drafting of this manuscript or its publication.

Figures created with Biorender.com

\section{Conclusion}

Individuals with $2 \mathrm{e}$ represent a diverse community with rich lives and extraordinary gifts, although they also face substantial challenges. Both the strengths and struggles of people with 2e often go unnoticed or underappreciated as an exceptional ability may conceal the signs of impairment, or a disability may obscure the signals of extraordinary potential. The knowledge and tools of neuroscience have the capacity to provide the understanding that will empower individuals with $2 e$ to utilize their talents and support their difficulties. Neurobiological study of plasticity at the level of circuits, cells, and molecules can provide the data to delineate the underpinnings of a variety of $2 \mathrm{e}$ manifestations. As a scientific community there is much to contribute from understanding not just how we suffer from psychiatric disorder but also how we excel despite considerable challenges. 


\section{References}

Reis, S. M., Baum, S. M., \& Burke, E. (2014). An operational definition of twice-exceptional learners. Gifted Child Quarterly, 58(3), 217-230. http://doi.org/10.1177/0016986214534976

National Education Association. (2006). The Twice-exceptional dilemma. Washington, D.C.

Assouline, S., Nicpon, M., \& Huber, D. (2006). The impact of vulnerabilities and strengths on the academic experiences of twice-exceptional students: A message to school counselors. Professional School Counseling, 10(1), 14-24.

http://doi.org/10.5330/prsc.10.1.y0677616t5j15511

Assouline, S. G., \& Whiteman, C. S. (2011). Twice-exceptionality: Implications for school psychologists in the post-IDEA 2004 era. Journal of Applied School Psychology, 27(4), 380-402. http://doi.org/10.1080/15377903.2011.616576

Beckmann, E., \& Minnaert, A. (2018). Non-cognitive characteristics of gifted students with learning disabilities: An in-depth systematic review. Frontiers in Psychology, 9. http://doi.org/10.3389/fpsyg.2018.00504

Foley-Nicpon, M., Assouline, S. G., \& Colangelo, N. (2013). Twice-Exceptional Learners: Who Needs to Know What? Gifted Child Quarterly, 57(3), 169-180. http://doi.org/10.1177/0016986213490021

Budding, D., \& Chidekel, D. (2012). ADHD and Giftedness: A Neurocognitive consideration of Twice exceptionality. Applied Neuropsychology: Child, 1(2), 145-151. http://doi.org/10.1080/21622965.2012.699423

Maddocks, D. L. (2019). Cognitive and achievement characteristics of students from a national sample identified as potentially twice exceptional (gifted with a learning disability). Gifted Child Quarterly, 64(1), 3-18.

http://doi.org/10.1177/0016986219886668

Cain, M. K., Kaboski, J. R., \& Gilger, J. W. (2019). Profiles and academic trajectories of cognitively gifted children with autism spectrum disorder. Autism, 23(7), 1663-1674. http://doi.org/10.1177/1362361318804019

Gardner, H. (1983). Frames of Mind: A Theory of Multiple Intelligences. New York, NY: Basic Books.

Sternberg, R. J. (1985). Beyond IQ: a triarchic theory of human intelligence. Cambridge, UK: Cambridge University Press.

Gilger, J. W., \& Hynd, G. W. (2008). Neurodevelopmental variation as a framework for thinking about the twice exceptional. Roeper Review, 30(4), 214-228. http://doi.org/10.1080/02783190802363893

Takeuchi, T., Duszkiewicz, A. J., \& Morris, R. G. (2014). The synaptic plasticity and memory hypothesis: encoding, storage and persistence. Philosophical Transactions of the Royal Society B: Biological Sciences, 369(1633), 20130288.

http://doi.org/10.1098/rstb.2013.0288

Brans, R. G., Kahn, R. S., Schnack, H. G., van Baal, G. C., Posthuma, D., van Haren, N. E., ... Hulshoff Pol, H. E. (2010). Brain plasticity and intellectual ability are influenced by shared genes. Journal of Neuroscience, 30(16), 5519-5524.

http://doi.org/10.1523/jneurosci.5841-09.2010

Bourgeron, T. (2015). From the genetic architecture to synaptic plasticity in autism spectrum disorder. Nature Reviews Neuroscience, 16(9), 551-563. http://doi.org/10.1038/nrn3992

Hansel, C. (2019). Deregulation of synaptic plasticity in autism. Neuroscience Letters, 688, 58-61.

http://doi.org/10.1016/j.neulet.2018.02.003

Sherry, D. F., \& Schacter, D. L. (1987). The evolution of multiple memory systems. Psychological Review, 94(4), 439-454. http://doi.org/10.1037/0033-295x.94.4.439

Squire, L. R. (1992). Declarative and Nondeclarative Memory: Multiple Brain Systems Supporting Learning and Memory. Journal of Cognitive Neuroscience, 4(3), 232-243. http://doi.org/10.1162/jocn.1992.4.3.232 
Scoville, W. B., \& Milner, B. (1957). Loss of recent memory after bilateral hippocampal lesions. Journal of Neurology, Neurosurgery \& Psychiatry, 20(1), 11-21. http://doi.org/10.1136/jnnp.20.1.11

McDonald, R. J., \& White, N. M. (1993). A triple dissociation of memory systems: Hippocampus, amygdala, and dorsal striatum. Behavioral Neuroscience, 107(1), 3-22. http://doi.org/10.1037/0735-7044.107.1.3

Bechara, A., Tranel, D., Damasio, H., Adolphs, R., Rockland, C., \& Damasio, A. (1995). Double dissociation of conditioning and declarative knowledge relative to the amygdala and hippocampus in humans. Science, 269(5227), 1115-1118. http://doi.org/10.1126/science.7652558

Ferbinteanu, J. (2019). Memory systems 2018 - Towards a new paradigm. Neurobiology of Learning and Memory, 157, 6178. http://doi.org/10.1016/j.nIm.2018.11.005

McDonald, R. J., \& White, N. M. (1995). Hippocampal and nonhippocampal contributions to place learning in rats. Behavioral Neuroscience, 109(4), 579-593. http://doi.org/10.1037/0735-7044.109.4.579

Bassett, D. S., \& Sporns, O. (2017). Network neuroscience. Nature Neuroscience, 20(3), 353-364.

http://doi.org/10.1038/nn.4502

Sinha, P., Kjelgaard, M. M., Gandhi, T. K., Tsourides, K., Cardinaux, A. L., Pantazis, D., ... Held, R. M. (2014). Autism as a disorder of prediction. Proceedings of the National Academy of Sciences, 111(42) 15220-15225.

https://doi.org/10.1073/pnas.1416797111

Nickl-Jockschat, T., Habel, U., Maria Michel, T., Manning, J., Laird, A. R., Fox, P. T., ... Eickhoff, S. B. (2012). Brain structure anomalies in autism spectrum disorder-a meta-analysis of VBM studies using anatomic likelihood estimation. Human Brain Mapping, 33(6), 1470-1489. http://doi.org/10.1002/hbm.21299

Janouschek, H., Chase, H. W., Sharkey, R. J., Peterson, Z. J., Camilleri, J. A., Abel, T., ... Nickl-Jockschat, T. (2021). The functional neural architecture of dysfunctional reward processing in autism. Neurolmage: Clinical, 31, 102700.

http://doi.org/10.1016/j.nicl.2021.102700

Ullman, M. T., \& Pullman, M. Y. (2015). A compensatory role for declarative memory in neurodevelopmental disorders. Neuroscience \& Biobehavioral Reviews, 51, 205-222. http://doi.org/10.1016/j.neubiorev.2015.01.008

Boucher, J., \& Mayes, A. (2012). Memory in ASD: Have we been barking up the wrong tree? Autism, 16(6), 603-611. http://doi.org/10.1177/1362361311417738

Boucher, J., Mayes, A., \& Bigham, S. (2012). Memory in autistic spectrum disorder. Psychological Bulletin, 138(3), 458-496. http://doi.org/10.1037/a0026869

Portman, E. C. (2006). A social competence intervention program for children with high functioning autism and Asperger's Syndrome: A qualitative study. The University of Texas at Austin.

Dichter, G. S., Richey, J. A., Rittenberg, A. M., Sabatino, A., \& Bodfish, J. W. (2011). Reward circuitry function in autism during face anticipation and outcomes. Journal of Autism and Developmental Disorders, 42(2), $147-160$. http://doi.org/10.1007/s10803-011-1221-1

Baron-Cohen, S. (2006). The hyper-systemizing, assortative mating theory of autism. Progress in NeuroPsychopharmacology and Biological Psychiatry, 30(5), 865-872. http://doi.org/10.1016/j.pnpbp.2006.01.010

Baron-Cohen, S., \& Lombardo, M. V. (2017). Autism and talent: the cognitive and neural basis of systemizing. Autism Spectrum Disorders, 19(4), 345-353. http://doi.org/10.31887/dcns.2017.19.4/sbaroncohen

Hebb, D. O. (1949). The organization of behaviour: a neuro-psychological theory. New York: Wiley. 
Martin, S. J., Grimwood, P. D., \& Morris, R. G. (2000). Synaptic Plasticity and Memory: An Evaluation of the Hypothesis. Annual Review of Neuroscience, 23(1), 649-711. http://doi.org/10.1146/annurev.neuro.23.1.649

Bliss, T. V., \& Lømo, T. (1973). Long-lasting potentiation of synaptic transmission in the dentate area of the anaesthetized rabbit following stimulation of the perforant path. The Journal of Physiology, 232(2), 331-356.

http://doi.org/10.1113/jphysiol.1973.sp010273

Ramón y Cajal, S., \& Azoulay, L. (1894). Les nouvelles idées sur la structure du système nerveux chez l'homme et chez les vertébrés. http://doi.org/10.5962/bhl.title.48561

Baltaci, S. B., Mogulkoc, R., \& Baltaci, A. K. (2018). Molecular Mechanisms of Early and Late LTP. Neurochemical Research, 44(2), 281-296. http://doi.org/10.1007/s11064-018-2695-4

Morris, R. G., Anderson, E., Lynch, G. S., \& Baudry, M. (1986). Selective impairment of learning and blockade of long-term potentiation by an N-methyl-D-aspartate receptor antagonist, AP5. Nature, 319(6056), 774-776.

http://doi.org/10.1038/319774a0

Giese, K. P., Fedorov, N. P., Filipkowski, R. K., \& Silva, A. J. (1998). Autophosphorylation at Thr286 of the alpha calciumcalmodulin kinase II in LTP and learning. Science, 279(5352), 870-873. http://doi.org/10.1126/science.279.5352.870

Abel, T., Nguyen, P. V., Barad, M., Deuel, T. A. S., Kandel, E. R., \& Bourtchouladze, R. (1997). Genetic Demonstration of a Role for PKA in the Late Phase of LTP and in Hippocampus-Based Long-Term Memory. Cell, 88(5), 615-626.

http://doi.org/10.1016/s0092-8674(00)81904-2

Nabavi, S., Fox, R., Proulx, C. D., Lin, J. Y., Tsien, R. Y., \& Malinow, R. (2014). Engineering a memory with LTD and LTP. Nature, 511(7509), 348-352. http://doi.org/10.1038/nature13294

Lovinger, D. M. (2010). Neurotransmitter roles in synaptic modulation, plasticity and learning in the dorsal striatum. Neuropharmacology, 58(7), 951-961. http://doi.org/10.1016/j.neuropharm.2010.01.008

Kim, J., Park, E.-K., Shim, K.-W., \& Kim, D. S. (2018). Hemispherotomy and Functional Hemispherectomy: Indications and outcomes. Journal of Epilepsy Research, 8(1), 1-5. http://doi.org/10.14581/jer.18001

Kliemann, D., Adolphs, R., Paul, L. K., Tyszka, J. M., \& Tranel, D. (2021). Reorganization of the social brain in individuals with only one intact cerebral hemisphere. Brain Sciences, 11(8), 965. http://doi.org/10.3390/brainsci11080965

Kandel, E. R. (2001). The Molecular Biology of Memory Storage: A Dialogue Between Genes and Synapses. Science, 294(5544), 1030-1038. http://doi.org/10.1126/science.1067020

Abel, T., \& Lattal, K. M. (2001). Molecular mechanisms of memory acquisition, consolidation and retrieval. Current Opinion in Neurobiology, 11(2), 180-187. http://doi.org/10.1016/s0959-4388(00)00194-x

Kandel, E. R. (2012). The molecular biology of memory: CAMP, PKA, CRE, CREB-1, CREB-2, and CPEB. Molecular Brain, 5(1), 14. http://doi.org/10.1186/1756-6606-5-14

Levenson, J. M., \& Sweatt, J. D. (2005). Epigenetic mechanisms in memory formation. Nature Reviews Neuroscience, 6(2), 108-118. http://doi.org/10.1038/nrn1604

Zovkic, I. B. (2021). Epigenetics and memory: An expanded role for chromatin dynamics. Current Opinion in Neurobiology, 67, 58-65. http://doi.org/10.1016/j.conb.2020.08.007

Fischer, A., Sananbenesi, F., Wang, X., Dobbin, M., \& Tsai, L.-H. (2007). Recovery of learning and memory is associated with chromatin remodelling. Nature, 447(7141), 178-182. http://doi.org/10.1038/nature05772 
Kwok, R. P., Lundblad, J. R., Chrivia, J. C., Richards, J. P., Bächinger, H. P., Brennan, R. G., ... Goodman, R. H. (1994). Nuclear protein CBP is a coactivator for the transcription factor CREB. Nature, 370(6486), 223-226.

http://doi.org/10.1038/370223a0

Gräff, J., \& Tsai, L.-H. (2013). Histone acetylation: molecular mnemonics on the chromatin. Nature Reviews Neuroscience, 14(2), 97-111. http://doi.org/10.1038/nrn3427

Guan, Z., Giustetto, M., Lomvardas, S., Kim, J.-H., Miniaci, M. C., Schwartz, J. H., ... Kandel, E. R. (2002). Integration of LongTerm-Memory-Related Synaptic Plasticity Involves Bidirectional Regulation of Gene Expression and Chromatin Structure. Cell, 111(4), 483-493. http://doi.org/10.1016/s0092-8674(02)01074-7

Bourtchuladze, R., Frenguelli, B., Blendy, J., Cioffi, D., Schutz, G., \& Silva, A. J. (1994). Deficient long-term memory in mice with a targeted mutation of the cAMP-responsive element-binding protein. Cell, 79(1), 59-68. http://doi.org/10.1016/00928674(94)90400-6

Jarome, T. J., \& Lubin, F. D. (2013). Histone lysine methylation: Critical regulator of memory and behavior. Reviews in the Neurosciences, 24(4). http://doi.org/10.1515/revneuro-2013-0008

Gupta, S., Kim, S. Y., Artis, S., Molfese, D. L., Schumacher, A., Sweatt, J. D., ... Lubin, F. D. (2010). Histone methylation Regulates memory formation. Journal of Neuroscience, 30(10), 3589-3599. http://doi.org/10.1523/jneurosci.3732-09.2010

Gupta-Agarwal, S., Franklin, A. V., DeRamus, T., Wheelock, M., Davis, R. L., McMahon, L. L., \& Lubin, F. D. (2012). G9a/glp histone lysine dimethyltransferase complex activity in the hippocampus and the entorhinal cortex is required for gene activation and silencing during memory consolidation. Journal of Neuroscience, 32(16), 5440-5453.

http://doi.org/10.1523/jneurosci.0147-12.2012

Peixoto, L., \& Abel, T. (2012). The role of histone acetylation in memory formation and cognitive impairments. Neuropsychopharmacology, 38(1), 62-76. http://doi.org/10.1038/npp.2012.86

Siu, M. T., \& Weksberg, R. (2017). Epigenetics of autism spectrum disorder. Advances in Experimental Medicine and Biology, 63-90. http://doi.org/10.1007/978-3-319-53889-1_4

Christopher, M. A., Kyle, S. M., \& Katz, D. J. (2017). Neuroepigenetic mechanisms in disease. Epigenetics \& Chromatin, 10(1). http://doi.org/10.1186/s13072-017-0150-4

Abel, T., Martin, K. C., Bartsch, D., \& Kandel, E. R. (1998). Memory suppressor genes: Inhibitory constraints on the storage of long-term memory. Science, 279(5349), 338-341. http://doi.org/10.1126/science.279.5349.338

Noyes, N. C., Phan, A., \& Davis, R. L. (2021). Memory suppressor genes: Modulating acquisition, consolidation, and forgetting. Neuron. http://doi.org/10.1016/j.neuron.2021.08.001

Lee, Y.-S., \& Silva, A. J. (2009). The molecular and cellular biology of enhanced cognition. Nature Reviews Neuroscience, 10(2), 126-140. http://doi.org/10.1038/nrn2572

Burns, A. M., \& Gräff, J. (2021). Cognitive epigenetic priming: Leveraging histone acetylation for memory amelioration. Current Opinion in Neurobiology, 67, 75-84. http://doi.org/10.1016/j.conb.2020.08.011

Boess, F. G., Hendrix, M., van der Staay, F.-J., Erb, C., Schreiber, R., van Staveren, W., ... Koenig, G. (2004). Inhibition of phosphodiesterase 2 increases neuronal cGMP, synaptic plasticity and memory performance. Neuropharmacology, 47(7), 1081-1092. http://doi.org/10.1016/j.neuropharm.2004.07.040

van der Staay, F. J., Rutten, K., Bärfacker, L., DeVry, J., Erb, C., Heckroth, H., ... Hendrix, M. (2008). The novel selective PDE9 inhibitor BAY 73-6691 improves learning and memory in rodents. Neuropharmacology, 55(5), 908-918.

http://doi.org/10.1016/j.neuropharm.2008.07.005 
Tang, Y.-P., Shimizu, E., Dube, G. R., Rampon, C., Kerchner, G. A., Zhuo, M., ... Tsien, J. Z. (1999). Genetic enhancement of learning and memory in mice. Nature, 401(6748), 63-69. http://doi.org/10.1038/43432

Carlezon Jr, W., Duman, R., \& Nestler, E. (2005). The many faces of CREB. Trends in Neurosciences, 28(8), 436-445. http://doi.org/10.1016/j.tins.2005.06.005

Yin, J. C. P., Del Vecchio, M., Zhou, H., \& Tully, T. (1995). CREB as a Memory Modulator: induced expression of a dCREB2 activator isoform enhances long-term memory in drosophila. Cell, 81(1), 107-115. http://doi.org/10.1016/00928674(95)90375-5

Chatterjee, S., Angelakos, C. C., Bahl, E., Hawk, J. D., Gaine, M. E., Poplawski, S. G., ... Abel, T. (2020). The CBP KIX domain regulates long-term memory and circadian activity. BMC Biology, 18(1). http://doi.org/10.1186/s12915-020-00886-1

Barrot, M., Olivier, J. D., Perrotti, L. I., DiLeone, R. J., Berton, O., Eisch, A. J., ... Nestler, E. J. (2002). CREB activity in the nucleus accumbens shell controls gating of behavioral responses to emotional stimuli. Proceedings of the National Academy of Sciences, 99(17), 11435-11440. http://doi.org/10.1073/pnas.172091899

Barrot, M., Wallace, D. L., Bolanos, C. A., Graham, D. L., Perrotti, L. I., Neve, R. L., ... Nestler, E. J. (2005). Regulation of anxiety and initiation of sexual behavior by CREB in the nucleus accumbens. Proceedings of the National Academy of Sciences, 102(23), 8357-8362. http://doi.org/10.1073/pnas.0500587102

Bućan, M., \& Abel, T. (2002). The mouse: genetics meets behaviour. Nature Reviews Genetics, 3(2), $114-123$. http://doi.org/10.1038/nrg728

Havekes, R., \& Abel, T. (2009). Chapter 1 Genetic Dissection of Neural Circuits and Behavior in Mus musculus. Advances in Genetics, 1-38. http://doi.org/10.1016/s0065-2660(09)65001-x

Silverman, J. L., Yang, M., Lord, C., \& Crawley, J. N. (2010). Behavioural phenotyping assays for mouse models of autism. Nature Reviews Neuroscience, 11(7), 490-502. http://doi.org/10.1038/nrn2851

Sjoberg, E. A. (2017). Logical fallacies in animal model research. Behavioral and Brain Functions, 13(1).

http://doi.org/10.1186/s12993-017-0121-8

Kennedy, D. (2004). The Old File-Drawer Problem. Science, 305(5683), 451-451.

http://doi.org/10.1126/science.305.5683.451

Kuechler, A., Zink, A. M., Wieland, T., Lüdecke, H.-J., Cremer, K., Salviati, L., ... Engels, H. (2014). Loss-of-function variants of SETD5 cause intellectual disability and the core phenotype of microdeletion 3p25.3 syndrome. European Journal of Human Genetics, 23(6), 753-760. http://doi.org/10.1038/ejhg.2014.165

Deliu, E., Arecco, N., Morandell, J., Dotter, C. P., Contreras, X., Girardot, C., ... Novarino, G. (2018). Haploinsufficiency of the intellectual disability gene SETD5 disturbs developmental gene expression and cognition. Nature Neuroscience, 21(12), 1717-1727. http://doi.org/10.1038/s41593-018-0266-2

Sessa, A., Fagnocchi, L., Mastrototaro, G., Massimino, L., Zaghi, M., Indrigo, M., ... Broccoli, V. (2019). SETD5 Regulates Chromatin Methylation State and Preserves Global Transcriptional Fidelity during Brain Development and Neuronal Wiring. Neuron, 104(2). http://doi.org/10.1016/j.neuron.2019.07.013

Hogeveen, J., Krug, M. K., Geddert, R. M., Ragland, J. D., \& Solomon, M. (2020). Compensatory hippocampal recruitment supports preserved episodic memory in autism spectrum disorder. Biological Psychiatry: Cognitive Neuroscience and Neuroimaging, 5(1), 97-109. http://doi.org/10.1016/j.bpsc.2019.08.009

Bireley, M., Languis, M., \& Williamson, T. (1992). Physiological uniqueness: A new perspective on the learning disabled/gifted child. Roeper Review, 15(2), 101-107. http://doi.org/10.1080/02783199209553477 
SPARK Consortium. (2018). SPARK: A US Cohort of 50,000 Families to Accelerate Autism Research. Neuron, 97(3):488-493. http://doi.org/10.1016/j.neuron.2018.01.015.

Kumar, V. J., Grissom, N. M., McKee, S. E., Schoch, H., Bowman, N., Havekes, R., ... Nickl-Jockschat, T. (2018). Linking spatial gene expression patterns to sex-specific brain structural changes on a mouse model of $16 \mathrm{p} 11.2$ hemideletion. Trans/ational Psychiatry, 8(1), 109. https://doi.org/10.1038/s41398-018-0157-z

Richiardi, J., Altmann, A., Milazzo, A. C., Chang, C., Chakravarty, M. M., Banaschewski, T., ... IMAGEN consortium (2015). BRAIN NETWORKS. Correlated gene expression supports synchronous activity in brain networks. Science, 348(6240), 12411244. https://doi.org/10.1126/science.1255905

Freel, B. A., Sheets, J. N., \& Francis, K. R. (2020). iPSC modeling of rare pediatric disorders. Journal of Neuroscience Methods, 332, 108533. http://doi.org/10.1016/j.jneumeth.2019.108533

Drakulic, D., Djurovic, S., Syed, Y. A., Trattaro, S., Caporale, N., Falk, A., ... Harwood, A. J. (2020). Copy number variants (CNVs): A powerful tool for IPSC-based modelling of ASD. Molecular Autism, 11(1). http://doi.org/10.1186/s13229-02000343-4 